\section{Effects of intertrial crossing punishment and d-amphetamine sulfate on avoidance and activity in four selectively bred rat strains*}

\author{
K. PAUL SATINDER \\ Lakehead University, Thunder Bay, Ont., Canada
}

The effects of punishing intertrial crossing (ITC) were investigated on avoidance response and shuttlebox activity in four selectively bred strains of rats, with and without d-amphetamine sulfate. In the Maudsley nonreactive strain (MNR/Har/Lu), the ITC punishment suppressed the avoidance response significantly, under both drug and nondrug conditions, but no such effects were found in the ilaudsley reactive (MR/Har/Lu) and the Roman high-avoidance (RHA/Lu) strains. However, d-amphetamine sulfate facilitated avoidance response in the $\mathrm{MR} / \mathrm{Har} / \mathrm{Lu}$ strain and suppressed it in the $\mathrm{RHA} / \mathrm{Lu}$. In the Roman low-avoidance strain (RLA/Lu), d-amphetamine sulfate tacilitated avoidance responding and activity, but in the ITC permissible group only.

Active avoidance performance of rats in a two-way shuttlebox may be affected by the spontaneous activity level of the animals, indicating some form of correspondence between avoidances and intertrial activity. This possibility has been indicated by the findings of Broadhurst \& Bignami (1965), Wilcock \& Broadhurst (1967), and Satinder (1968, 1971) showing significant positive correlations between the number of avoidance responses and frequency of intertrial crossings. This relationship is likely to become more evident when the avoidance response is investigated under the effects of drugs such as d-amphetamine sulfate and caffeine because CNS-stimulant drugs are known to induce increased general activity. Although Cole (1966, 1970) tried to give a possible explanation to such results, no systematic attempt has so far been made to investigate experimentally the basis for such a correspondence between avoidance responses and intertrial crossings.

This investigation was initiated by recent findings (Satinder, 1971) that pointed out that this relation between avoidance responses and intertrial crossings was strain dependent. In the Maudsley nonreactive (MNR/Har/Lu) and the Roman high-avoidance (RHA/Lu) strains of rats selectively bred for low-defecation score in open field and high rates of avoidance conditioning, respectively, this correlation was found both during training sessions as well as under the effects of d-amphetamine sulfate and caffeine. In the Maudsley reactive

*This research was supported in part by an operating research grant (APA-321) from the National Research Council of Canada to the author.
(MR/Har/Lu) strain, selectively bred for high-defecation score in open field, no such correlation was found under either of these conditions. And in the Roman low-avoidance (RLA/Lu) strain, selectively bred for low rates of avoidance conditioning, these correlations were absent during training sessions, but under the effects of the drugs they appeared to a certain degree. Details of these strains of rats have been reported in Satinder (1971).

This study attempted to investigate the correspondence between avoidance performance and intertrial activity by experimental manipulation of intertrial crossings, which was achieved either by permitting or by punishing the intertrial crossings, both with and without d-amphetamine sulfate.

\section{SUBJECTS}

Forty naive male rats, 10 from each strain of MNR/Har/Lu:S,o, $\mathrm{MR} / \mathrm{Har} / \mathrm{Lu}: \mathrm{S}_{35}$, RHA/Lu:S $\mathrm{S}_{20}$, and $\mathrm{RLA} / \mathrm{Lu}: \mathrm{S}_{20}$, were used as Ss. All the animals were $114 \pm 5$ days of age at the start of the experiment. All the animals were bred and reared in the laboratory and weaned at 28 days of age. The animals were maintained exactly in the same manner as reported earlier (Satinder, 1971).

\section{EXPERLMENTAL DESIGN}

The animals were given 50 training trials in blocks of 10 over 5 days to learn the avoidance response. The stimulus parameters were the same as those used in the previous investigation (Satinder, 1971). The conditioned stimulus was a $22 \cdot \mathrm{kHz}$ tone for $5 \mathrm{sec}$ at $82.5 \mathrm{~dB}$ of intensity against a background masking noise of $62.5 \mathrm{~dB}$ (sound intensities were averages at the floor level above the standard reference level of .0002 microbar, measured by General $R$ adio sound-level meter,
Type 1551-C). This was followed by an unconditioned stimulus (UCS) of electric shock for $5 \mathrm{sec}$. The shock generator was set for a pulse duration of $10 \mathrm{msec}$, with peak pulse amplitude of $9 \times 10^{3} \mathrm{~V}$ and peak pulse current of $3 \times 10^{-3} \mathrm{~A}$. The pulse repeated at the rate of $55 / \mathrm{sec}$. The intertrial interval was variable from 45 to $55 \mathrm{sec}$, with an average of $50 \mathrm{sec}$.

The animals of each strain were matched for their performance on the basis of the number of avoidance repsonses and frequency of intertrial crossings (ITC) made during the training period, and assigned to ITC-punishment or ITC-permissible groups at random. Although the "TTC condition" started in the second week, i.e., center portions of Fig. 1 and Fig. 2, the performance of the groups (formed later for the second and third weeks) during the training sessions is also shown (left portions of the figures) to give a clear view of any differences produced by the ITC conditions or by the administration of the drug. For practical reasons, the training trials were given from Monday through Friday, the "ITC condition" for both the groups, i.e., ITC punishment and ITC permissible, was carried out from Monday through Friday of the following week, and the effects of $d$-amphetamine sulfate on both the groups were studied during the third week, from Monday through Friday.

Five dosages of d-amphetamine sulfate, i.e., $0.5,1.0,2.0,3.0$, and $4.0 \mathrm{mg} / \mathrm{kg}$, were administered intraperitoneally (IP) in distilled water in the volume of $1 \mathrm{ml} / \mathrm{kg}$. Each animal received all five dosages on 5 different days, in different orders assigned at random.

A double-blind technique was used. The person testing the animals knew neither the experimental condition nor the strain.

\section{APPARATUS}

The apparatus was an automated shuttlebox without barrier, the details of which have been reported in a previous paper (Satinder, 1971). For this experiment, an automatic mechanism was added to the apparatus to administer electric shock for a certain duration of the same intensity as UCS, on intertrial crossings under the ITC-punishment condition. The shuttlebox was pivoted on its short axis in the center. When the animal crossed from one side to the other, the box tilted slightly and activated a microswitch.

\section{PROCEDURE}

Every day of the experiment, each animal was weighed and carried to the experimental room in a plastic cage. Approximately $1 \mathrm{~min}$ was permitted to adapt to the apparatus and then one 

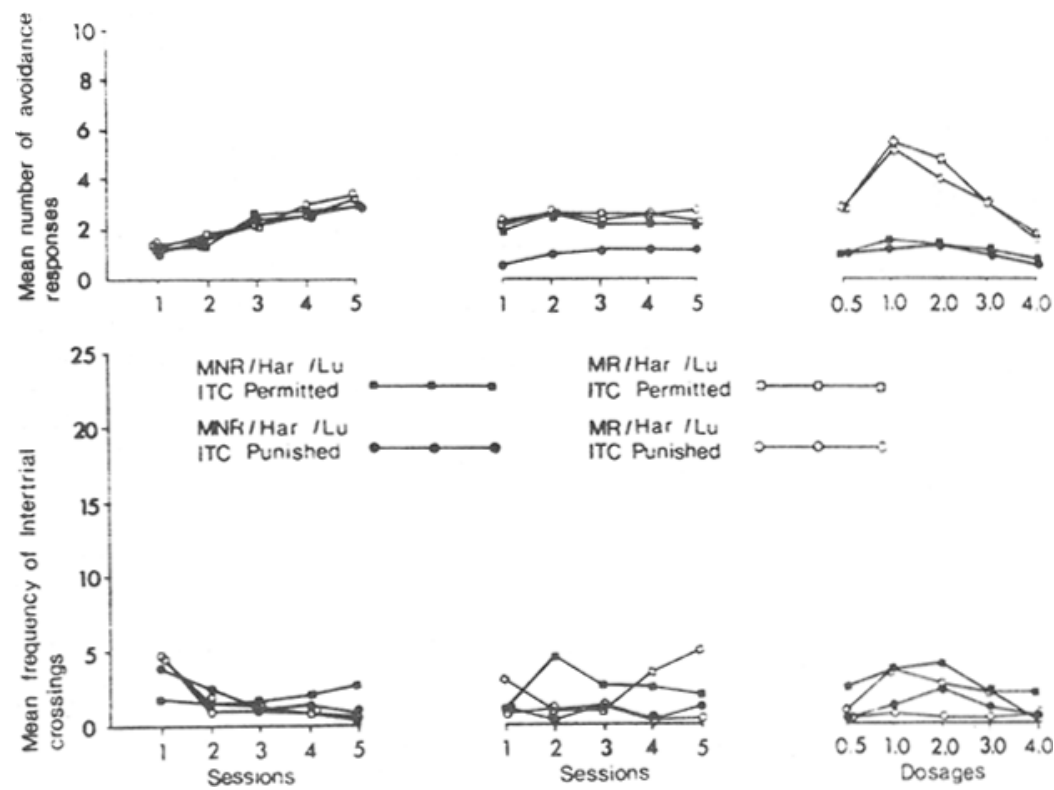

Fig. 1. Mean number of avoidance responses (upper portion) and mean frequency of intertrial crossings (lower portion) during training sessions (left), ITC condition sessions (center), and under the effects of d-amphetamine sulfate (right) of the MNR/Har/Lu and MR/Har/Lu strains of rats.

practice trial was given. After the practice trial, the apparatus was set for 10 trials for the day. After 10 trials were over, the animal was moved from the apparatus to its home cage. During the "ITC condition," the animals of the appropriate groups were either permitted intertrial crossings or received electric shock for $1 \mathrm{sec}$, of the same intensity as the UCS on each intertrial crossing. During the testing stage with $\mathrm{d}$-amphetamine sulfate, all the animals were injected (IP) with the assigned dosage, approximately at the same time of the day, $30 \mathrm{~min}$ before testing. The general procedure for the ITC conditions and the drug stage was the same as during the training period.

RESULTS AND DISCUSSION

The response measures of the mean number of avoidances and intertrial crossings were calculated and evaluated by analysis of variance. The results are presented in Fig. 1 for $\mathrm{MNR} / \mathrm{Har} / \mathrm{Lu}$ and $\mathrm{MR} / \mathrm{Har} / \mathrm{Lu}$ strains and in Fig. 2 for $\mathrm{RHA} / \mathrm{Lu}$ and RLA/Lu strains.

Avoidance Training

Figures 1 and 2 indicated that during the training sessions, all the strains except the RLA/Lu showed significant $(\mathrm{p}<.001)$ improvement in avoidance performance. It is also shown that there were no significant differences between MNR/Har/Lu and $\mathrm{MR} / \mathrm{Har} / \mathrm{Lu}$ strains; however, the RHA/Lu strain was significantly superior $(F=72.66, \mathrm{df}=1 / 16$, $p<.001)$ to the RLA/Lu strain. These findings are in line with the ones previously reported (Satinder, 1971). Effects of ITC Punishment

It is to be observed from Fig. 1 that the MNR/Har/Lu strain made significantly fewer avoidance responses $(\mathrm{F}=20.23, \mathrm{df}=1 / 8, \mathrm{p}<.005)$ and intertrial crossings $(p<.05)$ when the
ITCs were punished as compared to the ITC permissible condition. This may be interpreted to indicate that, in the nonreactive strain, the effects of ITC punishment resulted in response generalization to suppress activity both for avoidance responses and intertrial crossings. In the $\mathrm{MR} / \mathrm{Har} / \mathrm{Lu}$ strain, the ITC punishment did not affect the avoidance response at all; however, it did suppress the intertrial crossings $(p<.05)$ considerably. The $\mathrm{MNR} / \mathrm{Har} / \mathrm{Lu}$ animals performed at a significantly $(\mathrm{F}=19.96, \mathrm{df}=1 / 16$, $\mathrm{p}<005$ ) lower level of avoidance responses as compared to $\mathrm{MR} / \mathrm{Har} / \mathrm{Lu}$ animals in the ITC punishment groups, whereas these two strains did not differ on avoidance responses in the ITC permissible condition.

Figure 2 indicates that, in the RHA/Lu strain, avoidance responses were not affected by the ITC punishment, but intertrial crossings of this strain were significantly $(\mathrm{F}=$ $27.99, \mathrm{df}=1 / 8, \mathrm{p}<.005$ ) inhibited in the ITC punishment condition as compared to ITC permissible condition. In the RLA/Lu strain, there were practically no effects of the ITC punishment either on avoidance responses or on intertrial crossings. However, there is need of caution in the interpretation of the effects of ITC punishment in the low-avoidance (RLA/Lu) strain. The "no effect" here actually means "no decrease," since ITC punishment showed a decrease from higher levels of the response
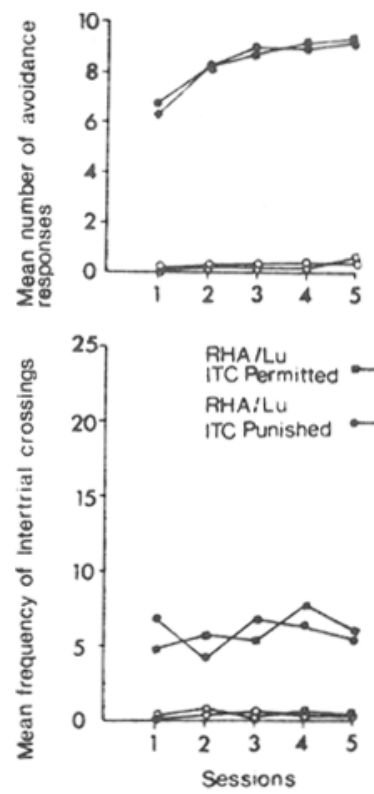

Fig. 2. Mean number of avoidance responses (upper portion) and mean frequency of intertrial crossings (lower portion) during training sessions (left), ITC condition sessions (center), and under the effects of d-amphetamine sulfate (right) of the RHA/Lu and RLA/Lu strains of rats. 
measures in other strains. The low-avoidance strain, however, is probably already as low on avoidance learning and intertrial activity as it can get, and so there is no further possibility of decrease. Hence, this "no effect" is probably at least in part due to baseline artifacts.

Effects of ITC Punishment and d-Amphetamine Sulfate

One must assume that avoidance learning was at an asymptote prior to the administration of $d$-amphetamine sulfate and also that the use of daily injections of the drug varying in dose from day to day did not have any carry-over effects. The analysis of days effects based on the number of avoidance responses verified that this assumption was in fact a fact and not an assumption in all the four strains of rats.

It is clear from Fig. 1 that d-amphetamine sulfate suppressed the avoidance responses in the ITC-permissible group of the $\mathrm{MNR} / \mathrm{Har} / \mathrm{Lu}$ strain of rats, and the drug did not have any effect on avoidance behavior in the ITC-punishment group. However, there was no particular effect of the drug on the intertrial crossings in either group. The overall inhibitory effect of the drug on avoidance responses in the nonreactive strain is in line with the earlier findings (Satinder, 1971).

In the $\mathrm{MR} / \mathrm{Har} / \mathrm{Lu}$ strain (Fig. 1), d-amphetamine sulfate induced significant facilitation $(p<.01)$ in avoidance responses in both the ITC permissible and punishment groups, but did not affect the intertrial activity in any group. The differential effects of ITC punishment and d-amphetamine sulfate on avoidance responses and intertrial activity indicate that there is no particular relationship between these two measures in the reactive strain and support the previous findings (Satinder, 1971). The enhancement of the avoidance responses under the effects of drug is similar to the earlier results (Satinder, 1971).

In the RHA/Lu strain (Fig. 2), d-amphetamine sulfate significantly suppressed $(p<.05)$ the avoidance responses in both ITC permissible and punishment groups. There was significant increase $(F=14.25, \mathrm{df}=$
1/8, $p<.01$ ) in intertrial crossings under the effects of the drug in ITC permissible group and no change in the TTC punisinment group. It seems that the inhibitory effects of d-amphetamine sulfate on avoidance responses is independent of ITC condition to a certain extent. However, the intertrial activity under the effects of the drug is very much dependerit on the ITC condition in this strain. In general, the effects of d-amphetamine sulfate on both these measures are in support of the previous findings (Satinder, 1971).

In the RLA/Lu strain (Fig. 2), d-amphetamine sulfate facilitated significantly the avoidances $(\mathrm{F}=$ $10.29, \mathrm{df}=1 / 8, \mathrm{p}<.025)$ and intertrial activity $(\mathrm{p}<.05)$ in the ITC permissible group. However, in the ITC punishment group, there was no change due to the drug effect either in the avoidance responses or in the intertrial activity. These findings confirm the previous observation (Satinder, 1971) that in the low-avoidance strain, the facilitation in avoidance behavior under the effects of d-amphetamine sulfate is confounded with an increase in general activity.

It may be noticed that in spite of the fact that there were some procedural variations with respect to the drug dosages used in this investigation, the overall drug effects on avoidance performance and intertrial activity are essentially the same as reported earlier (Satinder, 1971).

These findings clearly indicate that intertrial crossing punishment can produce strain-dependent dissociation between avoidance and intertrial responses. Furthermore, these observed dissociations are also influenced by d-amphetamine sulfate. In summary, in the nonreactive (MNR/Har/Lu) strain, the ITC punishment suppressed two-way active avoidance as well as intertrial activity and d-amphetamine sulfate did not affect either of the two response measures under the ITC-punishment condition, but under the ITC-permissible condition the drug inhibited the avoidance behavior and did not affect the intertrial activity. This indicates that ITC punishment did not produce any dissociation between the avoidances and intertrial activity, but that d-amphetamine sulfate did so under the ITC-permissible condition in this strain. In the reactive (MR/Har/Lu) strain, the ITC punishment suppressed only intertrial crossings and not avoidances, but the drug facilitated avoidances and did not affect intertrial activity under either of the two ITC conditions, showing that both ITC punishment and the drug induced dissociations in different directions between the two response measures. The high-avoidance (RHA/Lu) strain showed no effect of the ITC punishment oil avoidances but showed a clear suppression of activity. In this strain, the drug inhibited the avoidances under both ITC conditions but increased intertrial activity under the ITC-permissible condition and produced no effect under ITC punishment. This shows that ITC punishment and the drug both produced dissociations between the two measures, but in different directions. The low-avoidance (RLA/Lu) strain was not affected by the ITC punishment on either of the two measures, and, under the effects of drug, this strain showed facilitation in both avoidances and intertrial activity under the ITC-permissible condition only, showing no dissociations under any of the experimental conditions.

\section{REFERENCES}

BROADHURST, P. L., \& BIGNAMI, G. Correlative effects of psychogenetic selection: A study of the Roman high and low avoidance strains of rats. Behavior Research \& Therapy, 1965, 2, 273-280. COLE, S. O. Comments on the effects of amphetamine in avoidance conditioning. Psychological Reports, 1966, 19, 41-42.

COLE, S. O. Experimental effects of amphetamine: Supplementary report. Perceptual \& Motor Skills, 1970, 31. 223-232.

SATINDER, K. P. A note on the correlation between open-field and escape-avoidance behavior in the rat. The Journal of Psy chology, 1968, 69, 3-6.

SATINDER, $K$. P. Genoty pe-dependent effects of d-amphetamine sulphate and caffeine on escape-avoidance behavior of rats. Journal of Comparative \& Physiological Psychology, 1971, 76, 359-364.

WILCOCK, J. \& BROADHURST, P. L. Strain differences in emotionality: Open-field and conditioned avoidance behavior in the rat. Journal of Comparative \& Physiological Psychology, $1967,63,335-338$. 